\section{Inside Plant Cells}

Plant Cell Physiology: a Physicochemical Approach. By Park S. Nobel. (A Series of Books in Biology.) Pp. viii + 267. (Freeman: San Francisco and Reading, January 1971.) $£ 3.60$.

THIS book has been written primarily for advanced undergraduate and graduate students of plant physiology and biophysics. Although the author has presented a fairly rigorous physicochemical approach in discussing plant cell processes, the reader does require some previous knowledge of elementary physical chemistry and calculus. The title is a little misleading, for the contents are essentially restricted to Nobel's own specialities, namely ion and water movement in plant cells and primary energy conversion processes of photosynthesis. It does not, however, include extensive experimental observations, but stresses the theoretical concepts which govern these processes.

There are six chapters, the first of which consists of a mixture of topics ranging from the basic morphological features of plant cells to Fick's laws of diffusion. Fortunately, the contents of the successive chapters have been more logically thought out. In fact, the second chapter gives an excellent description of the properties of water and its movement in plant cells and their organelles, as well as considering briefly specialized plant tissue such as the xylem and roots. This topic has been approached in terms of classical thermodynamics and the reader is introduced to quantities such as chemical and water potential. This thermodynamic approach is continued in the following chapter, which is partly concerned with the principles governing the passive distribution in, and flux of charged solutes between, various compartments of plant cells. For example, the concepts which give rise to the derivation of such expressions as the Nernst equation, the Gold. man equation and the Ussing-Teorell equation are explained. This chapter on solutes also includes a short section on active transport and an introduction to the basic concepts of irreversible thermodynamics emphasizing particularly how reflexion coefficients can be used to modify classical equations such as the Boyle-Van't Hoff relation.

The remaining chapters are essentially concerned with energy conversion in plant cells, with particular reference to photosynthesis. A complete chapter is devoted to elementary photochemistry and, as such, gives a reasonable backing for the following chapter which deals with primary photosynthetic mechanisms. There is, for example, a detailed description of the optical and chemical properties of chlorophyll coupled to a section on excitation transfer and trapping. The remaining part of the chapter outlines current ideas on electron transport, but does not deal with the biochemistry of carbon fixation. Finally, the last chapter considers briefly the thermodynamics of both chloroplast and mitochondrial energetics.

As far as I am aware there is no other textbook available which covers these topics of plant physiology and also deals with their physicochemical aspects. Consequently Nobel has made available an excellent and needed textbook for those who teach in this field. The earlier chapters on water and solutes are particularly valuable, although the section on photosynthesis does nothing more than present information which can be readily found in other recently published textbooks specifically written on this subject. The value of the book for teaching purposes is increased by the numerical problems at the end of each chapter, which have been designed to give the student an opportunity to apply the principles and equations presented in the text.

J. BARBER

\section{Physiology of Emotion}

Physiological Correlates of Emotion. Edited by Perry Black. Pp. xvi +309 . (Academic: New York and London, October 1970.) $£ 6.30$.

THis book consists of seven papers delivered at a conference held in Baltimore, supplemented by six contributions invited by the editor. The list of contributors is impressive, with leading American experts covering a wide range of physiological and psychological aspects of the problem. It is perhaps surprising to note that there is no clinical psychiatrist contributing. This may be because none could be found with the necessary depth of neurophysiological knowledge to survive in this company. Nevertheless, it perhaps indicates the gap which at present exists between those involved in basic neurobiological research and the practitioners who are treating emotional disorders, and throws some doubt on the statement about the book made on the dust cover, ". . . it also serves as a useful stepping-stone toward the management and control of disordered emotional states". Papers such as those by McLean on "The Limbic Brain in Relation to the Psychoses" and Lindsley on "The Role of Nonspecific Reticulo-ThalamoCortical Systems in Emotion" should certainly narrow this gap. Both authors present a clear authoritative review of their subject and are also not afraid of speculation regarding the changes to be found in emotional disorders, thus providing a stimulus to further discussion and research. The paper by Harlow and Harlow on "Developmental Aspects of Emotional Behaviour" should also be singled out as a clear and stimulating review of original work in the field.

While other papers in the collection provide useful information and stimulating discussions, I felt that some of them, at times, had fallen into the trap of presenting too much in the way of detailed results which are to be found more appropriately in the papers of the appropriate scientific journal. In talking of emotion it seems that the contributors were most at home when considering arousal, fear and aggression. There is less material in the book dealing with depression or elation, although the contribution by Kety deals with some of the pharmacological aspects, and the paper by Dalgado on "Modulation of Emotions by Cerebral Radio Stimulation" also touches the subject.

In summary, the book provides a good introduction at a reasonably advanced level to the study of the physiology of emotion. The problems are not minimized and the reader should be stimulated to continue his own studies, realizing there is still much new country to be explored.

GEORGE W. AshCrofT

\section{Pictures Computed}

Computer Techniques in Image Processing. By Harry C. Andrews. (Academic: New York and London, 1970.) $\$ 10.50 ; £ 4.90$.

THE problems of transmission, storage and classification of pictures are receiving more and more attention from scientists and engineers. The analysis of the redundancies in the pictures is often complicated by noise, spurious distortions or translations. Physicists have known for a long time that real space is not always the most appropriate one for the analysis of phenomena: the achievements of quantum mechanics and electronic engineering are, to a great extent, derived from the idea that the phenomena are better studied if projected on a proper set of coordinates-time or frequency for electronic signals, space or momentum for quanta of energy. The extension of this concept to pictures is traditionally known as Fourier optics. Recently, the decomposition of pictures onto orthogonal functions other than sine and cosine has opened new horizons for this kind of technique. Particular interest has been shown in functions which can be implemented simply on digital computers.

The author, H. C. Andrews, and his collaborators, W. K. Pratt and K. Caspari, have been known for many years as productive contributors to the 